\title{
Application of Compensators for Non-Periodic Currents
}

\author{
Leon M. Tolbert ${ }^{1}$ \\ tolbert@utk.edu \\ ${ }^{1}$ Department of Electrical and Computer Engineering \\ The University of Tennessee \\ Knoxville, TN 37996-2100
}

\author{
Jianqing Chen ${ }^{1}$ \\ jchen5@utk.edu
}

\author{
Fang Z. Peng ${ }^{2}$ \\ fzpeng@msu.edu
}

\author{
John N. Chiasson ${ }^{1}$ \\ chiasson@utk.edu
}

\begin{abstract}
Based on a new definition of non-active current/power, this paper presents the application of a parallel active filter for the compensation of non-periodic currents. Analysis of the compensation characteristics required for a variety of non-periodic currents such as those associated with arc furnaces is presented. In addition, the corresponding current rating and energy storage requirements of the compensator are also presented.
\end{abstract}

Keywords - non-active power, compensator, non-periodic current, arc furnace, active filter

\section{INTRODUCTION}

Non-linear loads and power electronics converters are the most common sources of non-periodic currents and result in the distortion of voltage in power systems. Most power electronics converters generate harmonic components whose frequencies are integer multiples of the system fundamental frequency. However, in some cases, such as cycloconverters and line-commutated three-phase thyristor-based rectifiers, the line currents may contain both sub-harmonics (frequency lower than fundamental frequency) and super-harmonics (frequency higher than fundamental frequency but not an integer multiple of it). These waveforms are considered as non-periodic, although mathematically the currents may still have a periodic waveform, but in any event, the period of the currents is not equal to the period of the fundamental voltage [1], [2].

An arc furnace is another example of a non-linear load that generates non-periodic currents because it draws rapidly changing power from the source and the waveshape and amplitude are constantly changing. Voltage flicker and harmonic penetration problems associated with arc furnaces have been reported in several papers [3]-[5].

A transient disturbance may also be considered as one kind of non-periodic current from the compensation point of view. The disturbance may be caused by the sudden addition of a large load to the system such as starting a motor, a fault, or other reasons.

Definition and compensation of non-sinusoidal and nonperiodic currents and power has been a concern for some time [6]-[11]. However, most of the previous efforts have focused on the compensation of periodic non-sinusoidal currents
${ }^{2}$ Department of Electrical and Computer Engineering

Michigan State University

East Lansing, MI 48824-1226 instead of non-periodic currents. The diversity of the features of non-periodic currents makes their compensation quite difficult, and theoretically, their compensation is very different than that for periodic distorted currents. However, from a practical point of view, these two cases may be very close to each other [11]. Generally, in both cases, a sine wave with a constant rms magnitude is preferred for the source current after compensation.

If a conventional shunt active filter is used as the compensator, the compensator (in both cases) must inject all current components that are the difference between the desired source currents and the required load currents. Starting from this viewpoint, the work here characterizes the required compensation current, and in particular, determines the energy storage requirements for various non-periodic currents based on a new definition of non-active current [12].

\section{DEFINITION OF NON-ACTIVE CURRENT}

For a single or polyphase power system, a shunt compensator to minimize the nonactive power/current required of the source can be configured as in Fig. 1. It is assumed here that the shunt compensator consists only of passive components (inductor and/or capacitor) and/or switching devices and no external power source. As a consequence (neglecting the compensator's power loss), conservation of energy requires the active power of the compensator average zero. In more detail, let $p_{S}(t), p_{L}(t)$, and $p_{C}(t)$ denote the instantaneous power of the source, load and compensator, respectively, and their average values over a time interval $T_{C}$ are given by

$$
P_{X}(t)=\frac{1}{T_{C}} \int_{t-T_{C}}^{t} p_{X}(\tau) d \tau \text { where } X=S, L, \text { or } C .
$$

Then

$$
P_{S}(t)+P_{C}(t)=P_{L}(t)
$$

and

$$
P_{S}(t)=P_{L}(t), P_{C}(t)=0 \text { as } t \rightarrow \infty \text {. }
$$




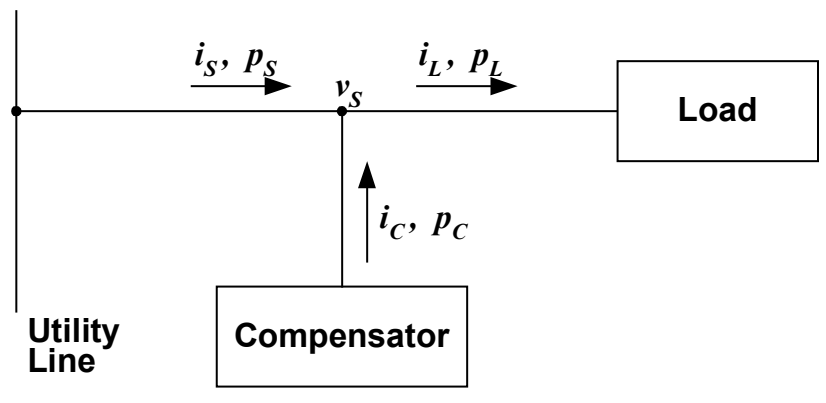

Fig. 1. A shunt compensator configuration.

In (1), $T_{C}$ is the averaging interval which can be zero, one fundamental cycle, one-half cycle, or multiple cycles, depending on the compensation objectives and the passive components' energy storage capacity. The subscripts " $S$ ", " $L$ ", and " $C$ " denote the source, load, and compensator quantities as shown in Fig. 1, respectively. Equations (1), (2) and (3) must hold true regardless of single-phase or polyphase, passive compensation or active compensation. Based on these physical and practical limitations, non-active power/current can be defined and formulated.

Here, Fryze's idea of non-active current/power [9] is extended by defining

$$
i_{p}(t)=\frac{P_{L}(t)}{V_{P}^{2}(t)} v_{P}(t), \quad i_{q}(t)=i(t)-i_{p}(t),
$$

where $v_{P}(t)$ is the reference voltage (see explanation below), $i_{p}(t)$ is the active current, $i_{q}(t)$ is the non-active current and

$$
V_{P}(t)=\sqrt{\frac{1}{T_{C}} \int_{t-T_{C}}^{t} v_{P}^{2}(\tau) d \tau} .
$$

$P_{L}(t)$ is the average active power over the interval $\left[t-T_{C}, t\right]$, which is calculated from (1). $V_{P}(t)$ is the rms value of the voltage calculated using $v_{P}(t)$ over the interval $\left[t-T_{C}, t\right]$ as in (5). Here $v_{P}(t)$ is the reference voltage whose specification depends on the compensation objectives. For example, this specification can be the terminal voltage $v_{s}(t)$ itself or it may be the fundamental component of $v_{s}(t)$ (i.e., $v_{P}(t)=v_{f}(t)$ where $v_{s}(t)=v_{f}(t)+v_{h}(t)$ and $v_{f}(t)$ is the fundamental and $v_{h}(t)$ is the harmonic component).

With the definition of current components $i_{p}(t)$ and $i_{q}(t)$ given above, $i_{p}(t)$ will provide the average active power $P_{L}(t)$ to the load during the interval $\left[t-T_{C}, t\right]$, and $i_{q}(t)$ provides no active power to the load. Therefore, $i_{C}(t)$ is chosen to be equal to $i_{q}(t)$ so that the compensator provides the entire nonactive current instead of the source.

The definitions (4) and (5) are valid for single- and polyphase circuits. However, in the case of polyphase circuits, the voltages and currents are expressed in vector form, which for a three-phase system is

$$
\begin{aligned}
& v=\left[v_{a}, v_{b}, v_{c}\right]^{T}, \\
& i=\left[i_{a}, i_{b}, i_{c}\right]^{T}, \text { and } \\
& v^{2}=\left[v_{a}, v_{b}, v_{c}\right] \cdot\left[v_{a}, v_{b}, v_{c}\right]^{T}=\left(v_{a}{ }^{2}+v_{b}{ }^{2}+v_{c}{ }^{2}\right) .
\end{aligned}
$$

It was shown in [12] that this new definition has the following features: 1) flexible to meet different compensation objectives; 2) valid for non-sinusoidal and non-periodic systems; 3) valid for single phase and polyphase systems. Table I illustrates that by choosing a different voltage reference or time averaging intervals, different source currents will result. Because of its flexibility in regards to compensation objectives, this definition is quite suitable for analyzing the compensation of non-periodic currents. This will be shown in the next section.

TABLE I. PARAMETERS FOR DIFFERENT COMPENSATION OBJECTIVES

\begin{tabular}{|l|c|c|l|}
\hline Compensation Objective & $\boldsymbol{v}_{\boldsymbol{P}}$ & $\boldsymbol{T}_{\boldsymbol{C}}$ & Resulting Source Current \\
\hline $\begin{array}{l}\text { Single-phase or polyphase } \\
\text { reactive current }\end{array}$ & $v$ & $T / 2$ or $T$ & $\begin{array}{l}\text { Unity pf and sinusoidal for } \\
\text { sinusoidal } v_{s}\end{array}$ \\
\hline $\begin{array}{l}\text { Single-phase or polyphase } \\
\text { reactive current and } \\
\text { harmonic current }\end{array}$ & $v_{f}$ & $T / 2$ or $T$ & $\begin{array}{l}\text { Unity pf and sinusoidal } \\
\text { regardless of } v_{s} \text { distortion }\end{array}$ \\
\hline $\begin{array}{l}\text { Instantaneous reactive } \\
\text { power for polyphase system }\end{array}$ & $v$ & $T_{C} \rightarrow 0$ & $\begin{array}{l}\text { Instantaneously unity pf for } \\
\text { polyphase system }\end{array}$ \\
\hline $\begin{array}{l}\text { Non-periodic disturbance } \\
\text { current }\end{array}$ & $v_{f}$ & $n T$ & $\begin{array}{l}\text { Reduced amplitude and near } \\
\text { sine wave with unity pf }\end{array}$ \\
\hline $\begin{array}{l}\text { Subharmonic current } \\
\text { sure }\end{array}$ & $v_{f}$ & $m n T / 2$ & $\begin{array}{l}\text { Pure sine wave or smoothed } \\
\text { sine wave with unity pf }\end{array}$ \\
\hline $\begin{array}{l}\text { Stochastic non-periodic } \\
\text { current }\end{array}$ & $v_{f}$ & $n T$ & $\begin{array}{l}\text { Smoothed sine wave with } \\
\text { unity pf }\end{array}$ \\
\hline
\end{tabular}

\section{COMPENSATION OF NON-PERIODIC CURRENTS}

For compensation of periodic currents with fundamental period $T$, choosing different $T_{C}$ alone does not change the source current characteristics. With reference to (5), the average rms value of a periodic quantity does not depend on the time averaging interval $T_{C}$ if it is an integer multiple of $T / 2$.

Theoretically, the time averaging interval can be chosen as any arbitrary value in the case of non-periodic currents. However, it is desirable for the interval to be an integer multiple of the line frequency period because of the desire that the source current be sinusoidal and have the same frequency as the source voltage frequency. In general, the period of the line voltage is not the same as the period of the quasi-periodic current $i_{p}(t)$, or there is no period in the case of a non-periodic load current. Thus, choosing different $T_{C}$ will result in quite different source currents and compensator currents. Simulation results of the compensation of three different kinds of non-periodic currents are given in the following subsections. 

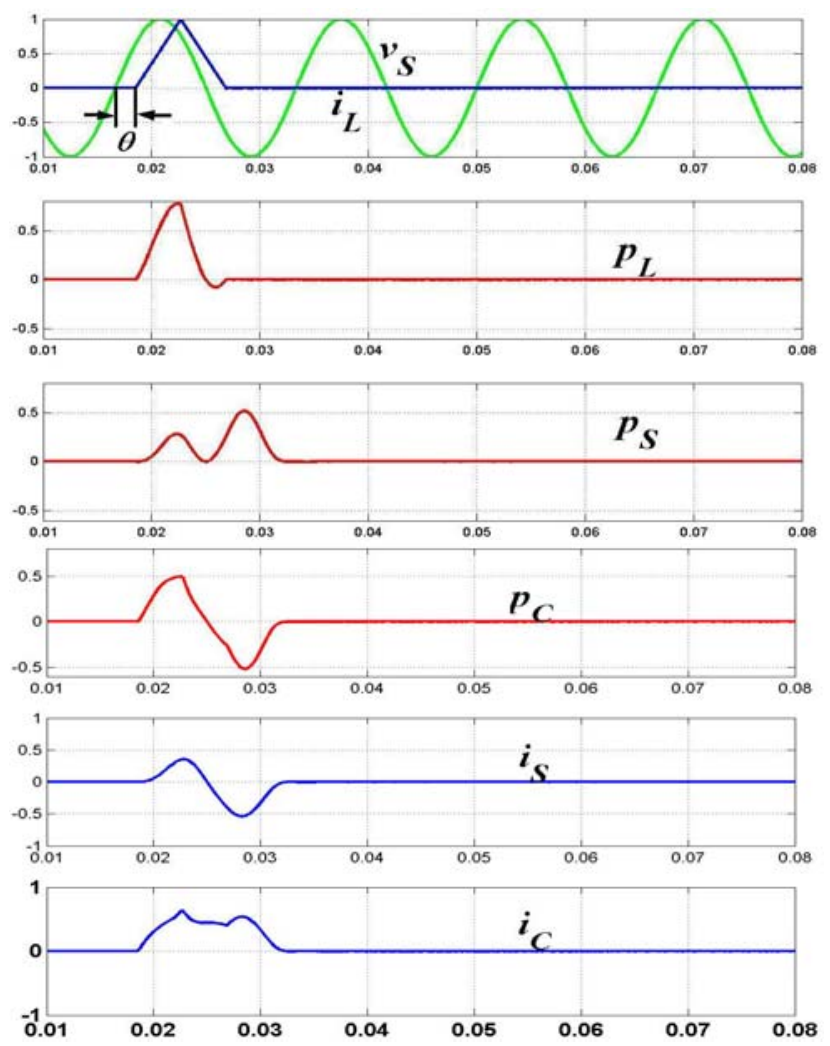

Fig. 2. Simulation results for disturbance type non-periodic current compensation $\left(T_{C}=T / 2\right)$.

\section{A. Non-periodic Disturbance Currents}

The duration of a non-periodic current may be a fraction of the line frequency cycle, or it may be several cycles. Outside this time period, the current may be zero or a pure sine wave. Figs. 2 and 3 show the simulation result of a single-phase disturbance current $i_{L}$ (pulse of duration that is $1 / 2$ of the voltage period) for two different compensation cases: $T_{C}=$ $T / 2$ (Fig. 2) and $T_{C}=2 T$ (Fig. 3), and $v_{P}=v_{f}$ for both cases. $\theta$ is the phase angle between the source voltage and beginning of the load current pulse, and in this case, $\theta=30^{\circ}$, as shown in Fig. 2 and Fig. 3. To simplify the simulation, the voltage waveform is assumed to be a sine wave. Note, however, that the result will also apply in the case of a source voltage that contains harmonics and is non-sinusoidal.

Because the disturbance energy is a fixed value, choosing a different time averaging interval $T_{C}$ results in different average active power $P_{L}$; thus, a different magnitude of source current and compensator current will result. Larger values of $T_{C}$ result in smaller peak values of $\left|i_{s}\right|$, i.e., the smaller disturbance seen from the source side. However, the compensator current rating will increase accordingly. Compensator design engineers must weigh the tradeoff between minimizing the source current against the cost of additional energy storage devices (capacitance/inductance).
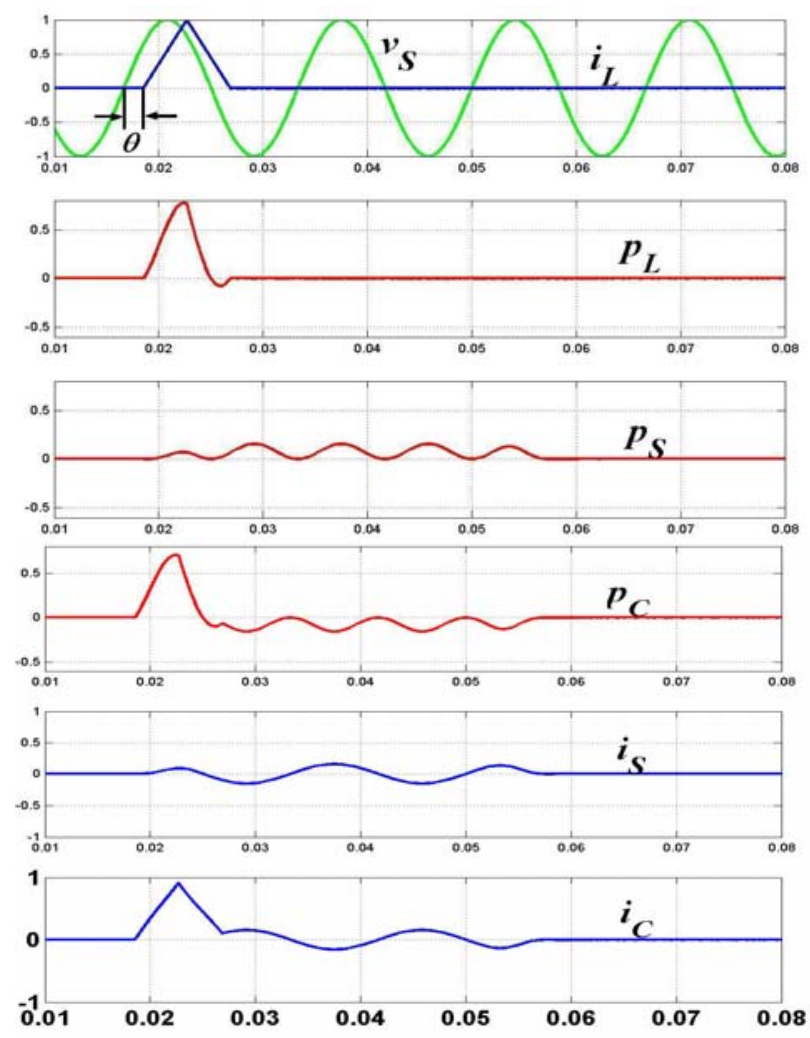

Fig. 3. Simulation results for disturbance type non-periodic current compensation $\left(T_{C}=2 T\right)$.

The simulations show that by increasing $T_{C}$ from $T / 2$ to $2 T$, the source current decreases significantly (from 0.6 p.u. to 0.2 p.u. of the load current), but the compensator current increases only slightly (from 0.6 p.u. to 0.9 p.u. of the load current). Thus, one can significantly decrease the source current with what may be a cost-effective increase in the compensator energy storage requirements. While these simulations are done for a single-phase case, the same result would be expected in three-phase cases.

\section{B. Subharmonic or Quasi-periodic Currents}

The main feature of this group of non-periodic currents is that the currents may have a repetitive period. The currents generated by power electronics converters may fall into this group. In the simulation shown in Fig. 4, the quasi-periodic current is composed by adding one subharmonic component $(12 \mathrm{~Hz})$ to the fundamental current $(60 \mathrm{~Hz})$, and the total harmonic distortion (THD) of load current is $26.7 \%$.

Figs. $4 \mathrm{~b}$ and $4 \mathrm{c}$ show the source current with compensation initiating at $\mathrm{t}=100 \mathrm{~ms}$. Here, $T c$ should be a multiple of $1 / 2$ of the common period of both the sub-harmonic and the fundamental if a sinusoidal source current is required. In the simulation in the paper, $T c$ should be $2.5 T, 5 T$, and so on, but can not be $3 T$ or $6 T$. For this case (Fig. $4 \mathrm{c}$ ), $T_{C}=2.5 T$ and the source current is a pure sine wave. Choosing $T_{C}$ smaller than 


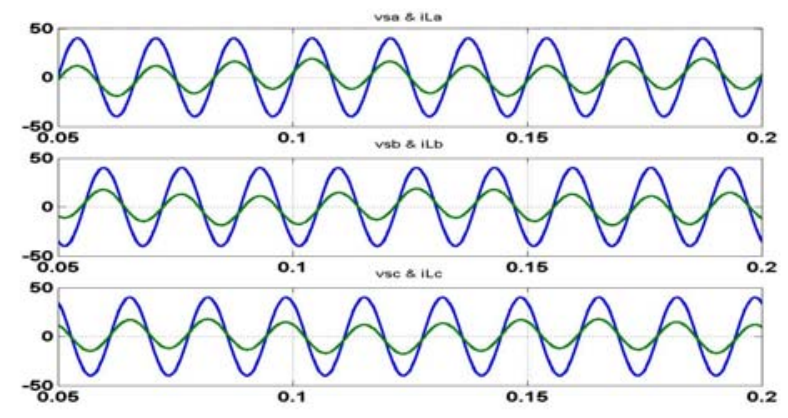

(a) 3-phase load current and voltage waveforms.

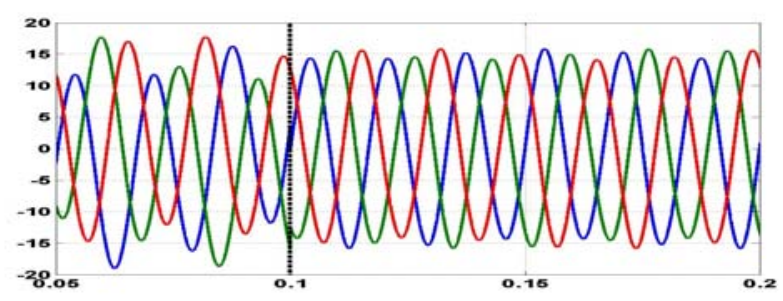

(b) Source currents compensated at $\mathrm{t}=100 \mathrm{~ms}\left(T_{C}=T\right)$

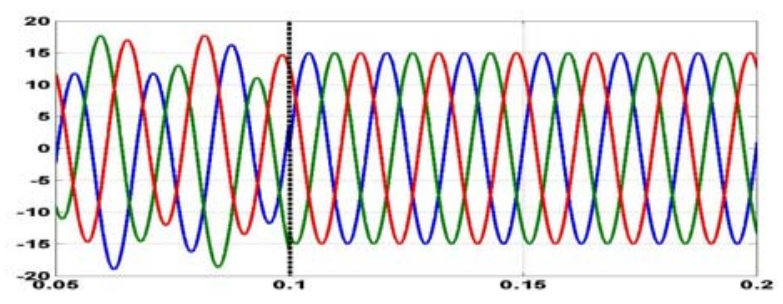

(c) Source currents compensated at $\mathrm{t}=100 \mathrm{~ms}\left(T_{C}=2.5 T\right)$

Fig. 4. Subharmonic current compensation.

$1 / 2$ of the common period will result in a source current that still contains some subharmonic components. When $T_{C}=T$, the THD of source current is $2.8 \%$ (Fig. $4 \mathrm{~b}$ ) because not all of the subharmonic component is eliminated; however, the source current THD has been reduced by $89.5 \%$ compared to the THD of the load current.

\section{Stochastic Non-Periodic Currents}

The load currents of arc furnaces are typically quite irregular as seen by the simulated arc furnace current shown in Fig. 5a. Thus, it is impossible to choose a specific $T_{C}$ to make the source current close to a sine wave. In the case of non-periodic waveforms, one can mathematically consider the period to be infinite. As can be seen from equations (3) and (4), as $T_{C}$ goes to infinity, both $P_{L}$ and $\mathrm{V}_{p}$ become constant and $i_{p}$ tracks the voltage reference $v_{p}$. If $v_{p}$ is chosen as $v_{f}$, then the source current will become sinusoidal. However, choosing the time interval to be infinite is not feasible in a practical application. But, it may still be possible to find some repetitive period in the current waveform that has most of the rms content of the waveform. Choosing that period as $T_{C}$ may get an acceptable source current which is quite close to a sine wave.

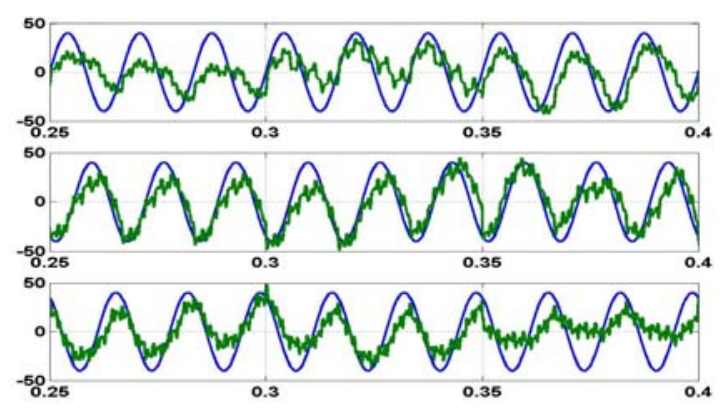

(a) 3-phase load current and voltage waveforms.

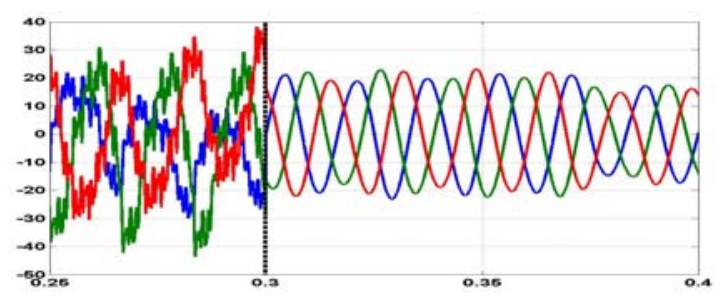

(b) Source currents compensated at $\mathrm{t}=300 \mathrm{~ms}\left(T_{C}=T\right)$

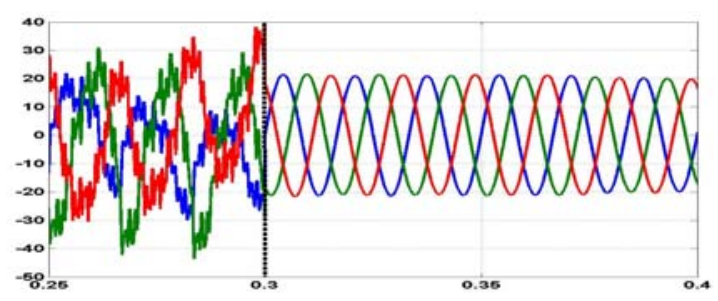

(c) Source currents compensated at $\mathrm{t}=300 \mathrm{~ms}\left(T_{C}=10 T\right)$

Fig. 5. Stochastic current compensation.

In Fig. 5a, the THD of the load currents $i_{L a}, i_{L b}$, and $i_{L c}$ are $75.7 \%, 47.5 \%$, and $52.7 \%$, respectively. When $T_{C}=T$ (Fig. 5b), the THD for each the source currents is $6.9 \%$ once compensation commences at $\mathrm{t}=300 \mathrm{~ms}$. All of the harmonics with frequencies that are an integral multiple of the fundamental frequency are completely eliminated. Harmonics with frequencies that are not an integral multiple of the fundamental frequency are also mitigated but are not completely compensated. However, when $T_{C}$ increases to $10 T$ (Fig. 5c), the source current is closer to a sine wave with a THD of $0.6 \%$. In this way, the voltage flicker and harmonic penetration problem [4], [5] associated with this non-periodic current waveform can be mitigated.

\section{ENERGY STORAGE REQUIREMENT OF COMPENSATOR}

The definition represented as (4) may be interpreted as the time average active power during $T_{C}$ that is contributed by the active source current $i_{p}$ where the average active power of the compensator $P_{C}(t)$ over $T_{C}$ is assumed to be zero.

$$
P_{C}(t)=\frac{1}{T_{C}} \int_{t-T c}^{t} v^{T}(\tau) i_{c} d \tau=0, i_{c}=i_{q}
$$




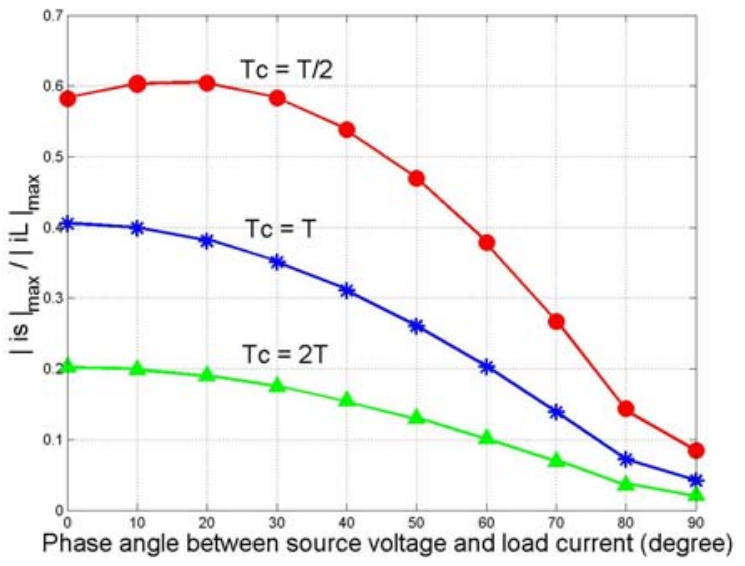

(a)

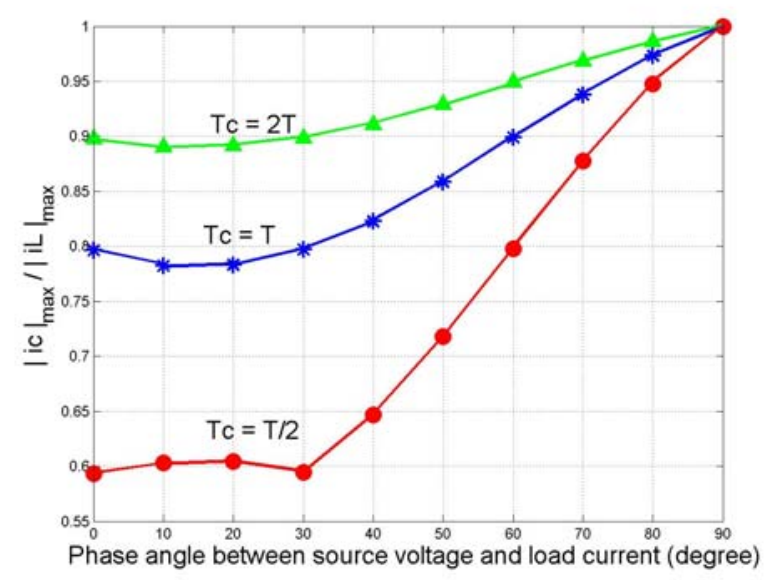

(b)

Fig. 6. (a) Peak source current and (b) peak compensator current normalized with respect to load current for different compensation times and load current phase angles.

It has been shown in [12] that the compensator average active power defined by (6) is zero. However, during the interval $T_{C}$, the instantaneous active $p_{C}(t)$ power is not necessarily zero. The capacitor is charged or discharged at different time intervals, and therefore a certain amount of energy storage is required. Different requirements will exist for energy storage by the active filter's capacitor to fulfill different compensation tasks. The maximum energy stored in the capacitance occurs at $t_{\max }$ when the capacitor changes from charge to discharge mode:

$$
\Delta E=\int_{0}^{t_{\max }} v^{T} i_{q} d t
$$

The choice of the time averaging interval $T_{C}$ is also significant in an energy storage design consideration. Choosing a longer $T_{C}$ results in a smoother source current with smaller amplitude; however, this requires that the

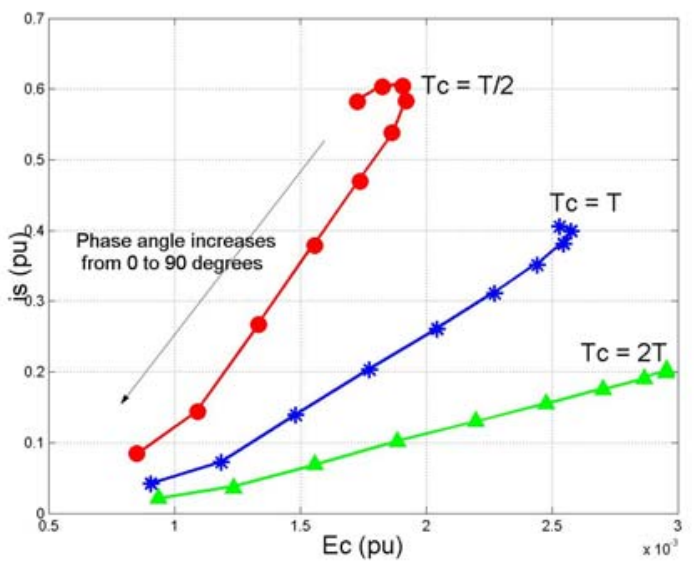

(a)

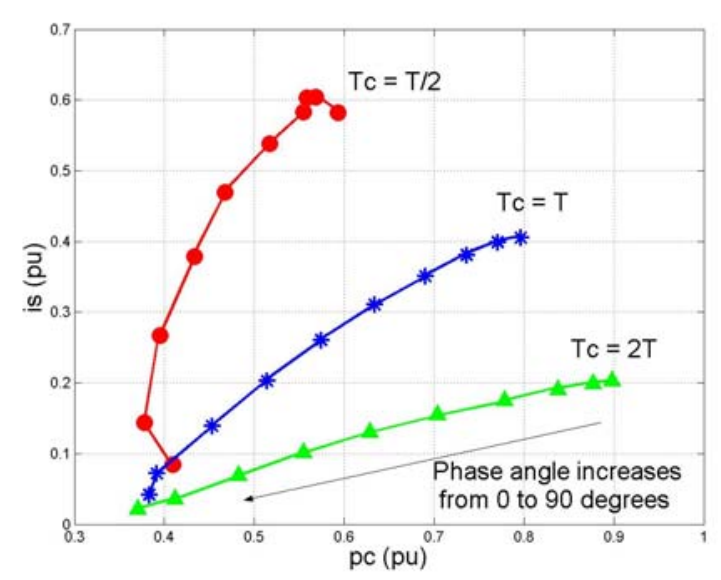

(b)

Fig. 7. Peak source current plotted as a function of the compensator's (a) energy storage requirement and (b) instantaneous power requirement for different compensation times and load current phase angles.

maximum required instantaneous compensator current $i_{c}$ increase as well as the energy storage requirement of the compensator.

Fig. 6 shows the peak source current normalized with respect to load current for various compensation intervals and phase angle $\theta$ between the initiation of the current pulse and source voltage for a triangular pulse waveform such as that shown in Figs. 2 and 3 . With the averaging interval $T_{C}$ changing from $T / 2$ to $2 T, i_{s}$ decreases from 0.6 p.u. to 0.2 p.u. of the load current while $i_{c}$ increases only modestly from 0.6 to 0.9 p.u. of the load current.

When the phase angle between the source voltage and pulse current is small, which is common for many systems, a small increase in energy storage capacity will result in a much better compensation (i.e., much smaller source current). In the case of a large phase angle difference with a compensation period of $2 T, i_{c}$ increases $10 \%$ (from 0.9 to 1.0 p.u. of the load current); while in the case of a compensation period of $T / 2, i_{c}$ increases approximately $70 \%$ (from 0.6 to 
1.0 p.u.). This is because if the compensator uses $T_{C}=2 T$, it must have a larger energy storage capacity (relative to using $\left.T_{\mathrm{C}}=T / 2\right)$ that is ready for not only a longer compensation interval, but also a larger instantaneous reactive component. Thus, a compensator with larger energy storage can obtain a better compensation and support a load that has a large reactive component.

Fig. 7 shows the peak source current plotted as a function of the compensator's energy storage requirement and instantaneous power requirement for various compensation times and load current phase angles. The energy storage requirement determines how much passive components (inductor and/or capacitor) are needed, whereas the instantaneous power requirement determines voltage and current ratings of the active devices to be used for the compensator.

At longer averaging intervals, the power drawn from the source is distributed during $T_{C}$ so that it has a smaller peak value instead of a short duration, high power pulse. Of course, the compensator instantaneous power requirement is more concentrated so that a higher energy rating is needed when the compensation duration is short. Over the complete compensation period, the compensator provides only reactive power and does not consume or generate any active power because the load energy is always provided by the source. Thus, a trade-off between a smoother source current waveform with lower amplitude and the size of the compensator should be considered when choosing the appropriate $T_{\mathrm{C}}$.

\section{CONCLuSIONS}

By combining a new non-active current definition and the conventional shunt active power filter, the application presented in this paper accomplishes the compensation of a variety of non-periodic currents in power systems. Simulation results give credibility to the applicability of the definition for a variety of load currents. According to different compensation cases and the goals to be achieved, different averaging time intervals for the compensator are chosen, which will determine the compensator's storage requirement and the extent of residual distortion in the source current.

\section{REFERENCES}

[1] E.H. Watanabe, M. Aredes, "Compensation of non-periodic currents using the instantaneous power theory," IEEE Power Engineering Society Summer Meeting, Seattle, USA, 2000, pp. 994-999.

[2] [2] L. S. Czarnecki, "Non-periodic currents: their properties, identification and compensation fundamentals," IEEE Power Engineering Society Summer Meeting, Seattle, USA, 2000, pp. 971976.

[3] G. Carpinelli, M. DiManno, et. al., "Ac and dc arc furnaces: a comparison on some power quality aspects," IEEE Power Engineering Society Summer Meeting, Edmonton, Canada, 1999, pp. 499-506.

[4] S. R. Mendis, M. T. Bishop, J. F. Witte, "Investigations of voltage flicker in electric arc furnace power systems," IEEE Industry Applications Magazine, vol. 2, no. 1, Jan./Feb. 1996, pp. 28-34.

[5] M. M. Morcos, J. C. Gomez, "Flicker sources and mitigation," IEEE Power Engineering Review, vol. 22, no. 11, Nov. 2002, pp. 5-10.

[6] H. Akagi, Y. Kanazawa, A. Nabae, "Instantaneous reactive power compensators comprising switching devices without energy storage components," IEEE Trans. Ind. Appl., vol. 20, May/June 1984, pp. 625-630.

[7] L. Rossetto, P. Tenti, "Evaluation of instantaneous power terms in multi-phase systems: techniques and application to power conditioning equipment," ETEP, vol. 4, no. 6, Nov./Dec. 1994.

[8] F. Z. Peng, J. S. Lai, "Reactive power and harmonic compensation based on the generalized instantaneous reactive power theory for threephase power systems," Proceedings of the 7th International Conference on Harmonics and Quality of Power, Las Vegas, USA, 1996, pp. 8389.

[9] L. M. Tolbert, T. G. Habetler, "Survey of active and non-active power definitions," IEEE International Power Electronics Congress, Acapulco, Mexico, 2000, pp. 73-79.

[10] H. Kim, F. Blaabjerg, B. Bak-Jensen, J. Choi, "Instantaneous Power Compensation in Three-Phase Systems by Using p-q-r Theory," IEEE Transactions on Power Electronics, vol. 17, no. 5, Sep. 2002, pp. 701710 .

[11] H. Akagi, "Active filters and energy storage systems operated under non-periodic conditions," IEEE Power Engineering Society Summer Meeting, Seattle, USA, 2000, pp. 965-970.

[12] F. Z. Peng, L. M. Tolbert, Z. Qian, "Definitions and compensation of non-active current in power systems," IEEE Power Electronics Specialists Conf., Cairns, Australia, 2002, pp. 1779-1784. 